\title{
Heparinas de bajo peso molecular frente a heparinas no fraccionadas en el tratamiento de la trombosis venosa profunda
}

\author{
J.J. MORENO-PALOMARES, R. M. FISAC-HERRERO*, A. HERRERO-DOMINGO, \\ E.M. FERREIRA-PASOS, J. GRASA**, D. REVERTE-CEJUDO
}

Servicios de Medicina Interna, Hematología* y Radiología**. Hospital General. Segovia

LOW-MOLECULAR-WEIGHT HEPARIN VERSUS UNFRACTIONA TED HEPARIN IN THE TREATMENT OF DEEP VEIN THROMBOSIS

\section{RESUMEN}

Antecedentes: Las heparinas de bajo peso molecular están sustituyendo de forma progresiva a las heparinas no fraccionadas en el tratamiento de la enfermedad tromboembólica, porque parecen haber demostrado ser seguras y eficaces.

Métodos: Para analizar su eficacia y seguridad en nuestro medio, se realizó un estudio prospectivo aleatorizado en el que se comparaba la heparina sódica con una heparina de bajo peso molecular en el tratamiento de las trombosis venosas profundas, diagnosticadas clínicamente y confirmadas por eco-Doppler. Se incluyeron 32 pacientes (21 varones y 11 mujeres) de los cuales 15 recibieron heparina sódica en perfusión continua y 17 dalteparina sódica, seguida posteriormente de dicumarínicos en todos los casos. Se realizó un seguimiento de un año.

Resultados: No hubo diferencias entre ambos grupos de tratamiento en eficacia clínica/radiológica a corto plazo ni en el año de seguimiento. Asimismo tampoco hubo diferencias significativas en cuanto a efectos secundarios.

Conclusión: En nuestro medio, las heparinas de bajo peso molecular resultan tan eficaces y seguras como la heparina sódica para el tratamiento de la trombosis venosa profunda, presentando una posología más cómoda para el paciente, evitando el uso de bombas de infusión y la práctica de controles analíticos, y posibilitando el tratamiento domiciliario.

PALABRAS CLAVE: Heparinas de bajo peso molecular. Tratamiento. Enfermedad tromboembólica.

\begin{abstract}
Background: Low-molecular-weight heparin $(L M W H)$ are replacing unfractionated heparin (UH) in treatment of deep vein thrombosis $(D V P)$, showing their security and effectiveness.

Methods: To demonstrate their security and effectiveness a randomi zed prospective study was realised. It compaired UH with LMWH in DP treatment, clinically diagnosed and confirmed with eco-Doppler. The study included 32 patients (21 males and 11 femeles); 15 received $U H$ in continuos i.v. infussion and 17 sodic dalteparine, with dicumarinic afterwards in every case. The patients were followed for one year.

Results: There were no differences between the two groups of treat ment in clinical/radiologycal effectiveness either in short term nor one year follow up. There were no significative differences in side effects.

Conclusion: LMWH are as effective and safe as UH for DVT treat ment. They also have a more confortable dosage for the patient, avoiding the i.v. infusion and the coagulation test, which allows treatment at home.
\end{abstract}

KEY WORDS: Low-molecular-weight heparin. Treatment. Thrombo embolic disease.

Moreno-Palomares JJ, Fisac-Herrero RM, Herrero-Domingo A, Ferreira-Pasos EM, Grasa J, Reverte-Cejudo D. Heparinas de bajo peso molecular frente a heparinas no fraccionadas en el tratamiento de la trombosis venosa profunda. An Med Interna (Madrid) 2001; 18: 364368.

\section{INTRODUCCIÓN}

El uso de las heparinas de bajo peso molecular (HBPM) en la profilaxis de la enfermedad tromboembólica está plenamente aceptado $(1,3)$, habiendo sustituido a la heparina no fraccionada (HNF) en dicha indicación, por presentar, como principal ventaja, una posología más cómoda, al administrarse en una o dos dosis al día vía subcutánea.
Cada vez son más los autores que preconizan el uso de las HBPM para el tratamiento de la enfermedad tromboembólica (ETE) (4-12), aunque existen ciertas reticencias y la Food and Drug Administration (FDA) americana aún no ha aprobado dicha indicación para todas las HBPM ni para todas las formas de ETE (trombosis venosa profunda y tromboembolismo pulmonar) (30). En el momento de redacción de este artículo sólo se encuentra aprobada por la FDA la enoxaparina para el

Trabajo aceptado: 24 de Enero de 2001

Correspondencia: J. Javier Moreno Palomares. Servicio de Medicina Interna. Hospital General. Carretera de Ávila, s/n. 40002 Segovia. 
tratamiento de la ETE (30). Esta situación ha hecho que en algunos foros se planteen las implicaciones legales que podrían derivar de su empleo sin dicha aprobación (12), así como del uso de otras HBPM. Son varias las HBPM, con propiedades farmacodinámicas distintas pero que parecen tener la misma eficacia clínica. Los ensayos realizados comparan distintas HBPM con placebo o con HNF, pero no una HBPM frente a otra, por lo que probablemente no se pueda seleccionar sólo una de ellas para su uso clínico.

Las HBPM presentan una serie de ventajas frente a las HNF en el tratamiento de la ETE, como son el hecho de que permiten una posología más cómoda con una $(6,14)$ o dos dosis diarias vía subcutánea, no precisan controles analíticos para ajustar la dosis, presentan menor número de complicaciones hemorrágicas graves $(4-7,27)$. Algunos trabajos señalan incluso que son más eficaces que las HNF por su mayor poder antiinflamatorio(15). En el metaanálisis de la Cochrane Library realizado por van der Belt y cols. (27), se encuentra además una menor mortalidad global en el grupo tratado con HBPM.

Otro aspecto importante del uso de las HBPM en el tratamiento de la ETE es el hecho de que en determinadas condiciones permite el tratamiento ambulatorio de este proceso, habiéndose realizado varios estudios que demuestran la seguridad de esta pauta (16-19), con la consiguiente mejora de la calidad de vida del paciente. Esto permite, además, un importante abaratamiento de costes derivados principalmente de la no-hospitalización $(20,21)$. Esta pauta se debe reservar a ETE de "bajo riesgo", y, además, se debe disponer de un seguimiento estrecho por parte de Atención Primaria. En cualquier caso sí que debe permitir acortar los ingresos hospitalarios por ETE. Además, incluso aunque los pacientes permanezcan ingresados el mismo tiempo que con las HNF, el hecho de una posología vía subcutánea sin necesidad de bombas de infusión continua intravenosa, sistemas de perfusión ni controles analíticos diarios para ajuste de dosis, hace que su empleo en pacientes ingresados sea también más económico.

Por todo ello nos planteamos realizar en nuestro medio un estudio prospectivo aleatorizado en el que comparamos la eficacia de las HBPM frente a las HNF para el tratamiento de las trombosis venosas profundas (TVP), así como sus efectos secundarios, con un seguimiento posterior de un año.

\section{MATERIAL Y MÉTODOS}

Planteamos un estudio prospectivo, durante un año en el que se incluyeron 32 pacientes ( 21 varones y 11 mujeres) diagnosticados de trombosis venosa profunda clínicamente y con confirmación por eco-Doppler. Se aleatorizaron de forma que 15 recibieron HNF (heparina sódica) y 17 HBPM (dalteparina sódica).

Antes de entrar en el estudio se excluyeron los casos con afectación exclusivamente poplitea o proximal a iliaca. Así mismo se excluyeron los casos secundarios a procesos neoplásicos o estados de hipercoagulabilidad conocidos, y aquellos que presentaban tromboembolismo pulmonar asociado.

La dalteparina sódica se usó en dosis de $200 \mathrm{U} / \mathrm{kg}$ de peso/24 h vía subcutánea. En caso de precisar más de 18.000 U/día se dividió la dosis total en dos, administradas cada 12 horas.

La dosis inicial de heparina sódica fue de $400 \mathrm{U} / \mathrm{kg}$ de peso/día vía intravenosa en perfusión continua, ajustada posteriormente según valores de tiempo de tromboplastina parcial activada (TTPA) de 1,5 a 2,5 el control inicial.

Se determinaron niveles plasmáticos de colesterol, triglicéridos, transaminasas, plaquetas, fibrinógeno, tiempo de protrombina y tiempo de tromboplastina parcial activada al diagnóstico y en los días cuatro y seis de tratamiento. Asimismo se realizó eco-Doppler al diagnóstico y a los seis días de tratamiento.

Se introdujo la anticoagulación oral con dicumarínicos al segundo día del ingreso y se mantuvo durante tres meses. Los pacientes permanecieron ingresados hasta alcanzar un INR de 2 a 2,5 durante dos días consecutivos, suspendiendo entonces las heparinas y siendo dados de alta para control ambulatorio.

Se realizó un seguimiento durante un año. Se repitió el eco-Doppler a los tres meses del diagnóstico, coincidiendo con la retirada de la anticoagulación oral, y revisión clínica a los tres, seis y doce meses, con atención a las recidivas y la existencia de síndrome postflebítico. Para la comparación de la evolución radiológica por eco-Doppler a los tres meses del diagnóstico, se clasificaron en "mejor", "igual" o "peor", según el criterio del ecografista, que desconocía el tratamiento usado. El síndrome postflebítico se evaluó por la presencia o ausencia de dolor, diferencia de diámetro en el miembro afecto de más de $2 \mathrm{~cm}$, cambio de coloración y edema, catalogándolo como "leve" cuando existía sólo un dato, "moderado" con dos, "severo" con tres y "muy severo" con los cuatro parámetros evaluados.

Para el estudio estadístico se utilizaron la Chi cuadrado para variables categóricas y la t de Student para variables cuantitativas continuas. Se consideraron significativos valores de $\mathrm{p}<0,05$.

\section{RESULTADOS}

De los 32 pacientes incluidos, 21 eran varones y 11 mujeres. La media de edad fue de 69 años, con un rango de 27 a 84 (Tabla I).

La localización de la TVP fue femoral en 16 casos (50\%), femoropoplitea en $13(40,6 \%)$, femoroiliaca en $2(6,3 \%)$ e ilíaca en 1 caso $(3,1 \%)$ (Tabla I).

\begin{tabular}{|c|c|c|}
\hline \multicolumn{3}{|c|}{ TABLA I } \\
\hline \multicolumn{3}{|c|}{$\begin{array}{l}\text { CARACTERÍSTICAS DEM O GRÁFICASY LO CALIZACIÓN DE } \\
\text { LA TVP (N=32) }\end{array}$} \\
\hline & HNF (15) & HBPM (17) \\
\hline Sexo (M ujer, Hombre) & $6 \mathrm{M}, 9 \mathrm{H}$ & $5 \mathrm{M}, 12 \mathrm{H}$ \\
\hline Edad (media) & 63 años & 70 años \\
\hline $\begin{array}{l}\text { Localización } \\
\text { TVP } \\
\text { - Femoral } \\
\text { - Femoropoplítea } \\
\text { - Femoroilíaca } \\
\text { - Ilíaca }\end{array}$ & $\begin{array}{c}6 / 15 \\
(40 \%) \\
8 / 15 \\
(53,3 \%) \\
1 / 15 \\
(6,7 \%) \\
0 / 15 \\
(0 \%)\end{array}$ & $\begin{array}{c}10 / 17 \\
(58,8 \%) \\
5 / 17 \\
(29,4 \%) \\
1 / 17 \\
(5,9 \%) \\
1 / 17 \\
(5,9 \%)\end{array}$ \\
\hline
\end{tabular}


A los seis días de tratamiento, de los 17 pacientes tratados con HBPM, 7 presentaban un eco-Doppler "mejor" y 10 "igual". En el grupo de HNF, 3 mostraban un eco-Doppler "mejor", 10 "igual" y en 2 casos no se pudo realizar el control ecográfico. En ninguno de los dos grupos hubo una evolución ecográfica a "peor". Al comparar el tipo de tratamiento (HBMP y HNF) y la evolución ecográfica ("mejor" o "igual") no hubo diferencias estadísticamente significativas, si bien observamos cierta tendencia a la evolución a "mejor" en el grupo tratado con HBPM (7/17 frente a 3/13) (Tabla II).

TABLA ॥

\begin{tabular}{ccc}
\multicolumn{3}{c}{ TABLA II } \\
EVO LUCIÓ N ECO - DO PPLER A LOS SEIS DÍAS DE \\
TRATAM IENTO SEGÚN EL TIPO DE TRATAM IENTO \\
$(\mathrm{N}=30) \mathrm{P}=0,9148$ \\
\hline \\
$\mathrm{HNF}(13)$ & $\mathrm{HBPM}(17)$ \\
\hline "M ejor" & $3 / 13$ & $7 / 17$ \\
& $(23 \%)$ & $(58,8 \%)$ \\
"Igual" & $10 / 13$ & $10 / 17$ \\
& $(78 \%)$ & $(41,2 \%)$
\end{tabular}

De la misma manera, al comparar la evolución clínica, en ninguno de los dos grupos progresó la TVP, y no hubo diferencias significativas entre ambos según el tipo de tratamiento.

Respecto a los parámetros hematológicos y bioquímicos entre el día 1 y el día 6 de tratamiento, no hubo modificaciones relevantes.

En ninguno de los dos grupos, a los seis días de tratamiento, hubo complicaciones hemorrágicas graves ni efectos adversos significativos.

El seguimiento posterior, a los tres meses del evento y al suprimir el tratamiento anticoagulante, se realizó en 27 pacientes, perdiéndose 5 casos. El control de eco-Doppler en esta fase mostraba 22 casos "mejor" y 5 "igual", ninguno "peor". Al analizar estos datos por grupos de tratamiento, no hubo diferencias entre uno y otro tratamiento (Tabla III).

En la evaluación del síndrome postflebítico, a los tres meses estaba ausente en 15 casos (55,5\%), "leve" en 4 $(14,8 \%)$, "moderado" en $6(22,2 \%)$ y "severo" y "muy severo" uno en cada uno $(3,7 \%)$ (Tabla IV).

A los seis meses del evento se pudo seguir a 25 pacientes,

\section{TABLA III}

EVOLUCIÓN ECO -DO PPLER A LOS TRES M ESES DE TRATAM IENTO SEGÚN EL TIPO DE TRATAM IENTO $(\mathrm{N}=27) \mathrm{P}=0,9268$

\begin{tabular}{lcc}
\hline & $H N F(13)$ & HBPM (14) \\
\hline "M ejor" & $11 / 13$ & $11 / 14$ \\
& $(84,6 \%)$ & $(78,6 \%)$ \\
"Igual" & $2 / 13$ & $3 / 14$ \\
& $(15,4 \%)$ & $(21,4 \%)$ \\
\hline
\end{tabular}

TABLA IV

SÍNDRO M E POSTFLEBÍTICO A LOS TRES M ESES SEGÚN EL TIPO DE TRATAM IENTO ( $N=27) \mathrm{P}=0,7298$

\begin{tabular}{lcc}
\hline & HNF (13) & HBPM (14) \\
\hline "Ausente" & $7 / 13(53,8 \%)$ & $8 / 14(57,2 \%)$ \\
"Leve" & $2 / 13(15,4 \%)$ & $2 / 14(14,3 \%)$ \\
"M oderado" & $3 / 13(23,1 \%)$ & $3 / 14(21,4 \%)$ \\
"Severo" & $0 / 13(0 \%)$ & $1 / 14(7,1 \%)$ \\
"M uy severo" & $1 / 13(7,7 \%)$ & $0 / 14(0 \%)$ \\
\hline
\end{tabular}

de los cuales 14 (56\%) no presentaban síndrome postflebítico, 5 (20\%) "leve", 2 (8\%) "moderado", 3 (12\%) "severo" y sólo 1 (4\%) "muy severo" (Tabla V).

$\mathrm{Al}$ año se evaluaron 22 pacientes del total, de los cuales 16 $(72,7 \%)$ no presentaban síndrome postflebítico, $5(22,7 \%)$ "leve, $1(4,5 \%)$ "moderado", y ninguno grave o muy grave. (Tabla VI).

Cuando se analizó la existencia de síndrome postflebítico a los tres, seis y doce meses, según el tratamiento empleado, no hubo diferencias significativas entre uno y otro grupo (Tablas IV, V y VI).

\section{TABLA V}

SÍNDRO M E POSTFLEBÍTICO A LOS SEIS M ESES SEGÚN EL TIPO DE TRATAM IENTO $(\mathrm{N}=25) \mathrm{P}=0,8274$

\begin{tabular}{lcc}
\hline & HNF (12) & HBPM (13) \\
\hline "Ausente" & $7 / 12(58,4 \%)$ & $7 / 13(53,8 \%)$ \\
"Leve" & $2 / 12(16,7 \%)$ & $3 / 13(23,1 \%)$ \\
"M oderado" & $1 / 12(8,3 \%)$ & $1 / 13(17,7 \%)$ \\
"Severo" & $1 / 12(8,3 \%)$ & $2 / 13(15,4 \%)$ \\
"M uy severo" & $1 / 12(8,3 \%)$ & $0 / 13(0 \%)$ \\
\hline
\end{tabular}

Durante el seguimiento se detectaron dos recidivas, ambas en pacientes que fueron tratados con HNF. Asimismo se diagnosticó después del inicio del estudio enfermedad neoplásica en tres casos $(9,3 \%)$, siendo estos un cáncer de páncreas, un cáncer gástrico y un melanoma. El diagnóstico de los tres procesos se produjo en los cuatro primeros meses del episodio trombótico, y en todos la enfermedad tumoral estaba diseminada al diagnóstico.

Se produjo una muerte no esperada por probable tromboembolismo pulmonar masivo en un paciente que estaba siendo tratado con HNF.

\section{TABLA VI}

SÍNDRO M E POSTFLEBITTICO A LOS DOCE M ESES SEGÚN EL TIPO DE TRATAM IENTO ( $N=22)$ AGRUPANDO VARIABLES "SEVERO " $Y$ “ MUY SEVERO" $P=0,5276$

\begin{tabular}{lcc}
\hline & HNF (10) & HBPM (12) \\
\hline "Ausente" & 7/10(70\%) & $9 / 12(75 \%)$ \\
"Leve" & $2 / 10(20 \%)$ & $3 / 12(25 \%)$ \\
"M oderado" & $1 / 10(10 \%)$ & $0 / 12(0 \%)$ \\
"Severo" & $0 / 10(0 \%)$ & $0 / 12(0 \%)$ \\
"M uy severo" & $0 / 10(0 \%)$ & $0 / 12(0 \%)$ \\
\hline
\end{tabular}




\section{DISCUSIÓN}

El estudio realizado nos demuestra, de acuerdo con otros estudios publicados, que las HBPM resultan al menos tan eficaces y seguras como las HNF en el tratamiento de las TVP $(4-7,27)$. En nuestro trabajo hemos usado la dalteparina sódica, pero sin duda esto es un efecto de grupo, ya que los distintos ensayos se han realizado con diferentes HBPM con resultados similares. Las HBPM poseen diferentes características farmacodinámicas, y no son intercambiables una por otra cuando se han comenzado a usar, pero su utilidad y eficacia clínica parecen similares. No obstante, la aprobación para su uso en distintos cuadros clínicos se hace de forma progresiva y para una de ellas, derivado del hecho de que dicha aprobación se realiza en función de determinados ensayos clínicos que usan una HBPM en concreto. No hemos encontrado ningún estudio en el que se compare una HBPM con otra.

Algunos trabajos han apuntado incluso que las HBPM resultan más eficaces que la HNF para el tratamiento de la ETE (27), sugiriendo que pueda deberse a su mayor poder antiinflamatorio (15). En nuestro estudio también hemos apreciado cierta tendencia a una mejor evolución a corto plazo con la HBPM. En cualquier caso esta diferencia no ha sido significativa.

Respecto a efectos secundarios, en nuestro trabajo no han aparecido complicaciones relevantes con ninguno de los dos tratamientos empleados. En otros estudios más amplios, las HBPM han presentado menos complicaciones hemorrágicas graves que las HNF (4-7,27).

En la evolución a largo plazo, con un seguimiento de hasta un año, los resultados han sido similares para uno y otro grupo, tanto en la evolución radiológica como en la aparición del síndrome posflebítico.

En cuanto a las recidivas, los datos son semejantes a los descritos en otros trabajos, pero dado lo pequeño de la muestra, no podemos sacar conclusiones con nuestro estudio. Igualmente, el diagnóstico de neoplasia después de la TVP lo hemos encontrado en una proporción similar a la descrita en la literatura, y de la misma manera, en los primeros meses del diagnóstico de TVP y como enfermedad diseminada $(28,29)$.

La limitación fundamental de nuestro trabajo es lo reducido de la muestra. No obstante, nuestros datos concuerdan con los de otras series $(4-7,27)$, como consecuencia de las cuales se está recomendando el uso de HBPM.

Ante la igualdad (si no superioridad) de eficacia y efectos secundarios de las HBPM frente a las HNF, el beneficio fundamental de aquellas radica en dos pilares. Por un lado son más cómodas de usar ya que su posología es vía subcutánea en una o dos dosis ajustadas según peso, evitando la necesidad de la infusión intravenosa continua que supone el uso de la heparina sódica. Ello debe derivar en una mejora de la calidad de vida del paciente durante el tratamiento de la fase aguda. Por otro lado, supone un abaratamiento de costes, derivado de lo innecesario de controles analíticos diarios para ajustar la dosis de heparina sódica, bombas y sistemas de infusión, que superan a la diferencia de precio entre la HBPM y la HNF a favor de esta última $(20,21)$.

Pero hay algo más. El hecho del uso del las HBPM vía subcutánea permite el tratamiento domiciliario de las TVP, y en este sentido se han realizado varios estudios con buenos resultados (16-19). Esto sí que supone una auténtica mejoría de la calidad de vida y un muy importante abaratamiento de costes al evitar el ingreso hospitalario. Probablemente el tratamiento domiciliario de estos procesos no es posible en todos los casos, y ello se debe valorar. Si optamos por esta actitud debemos asegurarnos que exista un adecuado cumplimiento, lo que probablemente suponga un apoyo familiar y una vigilancia por parte de Atención Primaria. Además, no debemos olvidar que el tratamiento se debe continuar con anticoagulantes orales, y, por supuesto, que la decisión de establecer el tratamiento requiere un diagnóstico de certeza previo, ya que estamos hablando de un tratamiento prolongado y no exento de complicaciones potencialmente graves. Una alternativa al ingreso hospitalario completo puede ser un ingreso corto de 24-48 horas que permita iniciar y programar el uso de anticoagulantes orales así como aleccionar al paciente sobre el empleo de los mismos y de las HBPM durante el tiempo que deban usarlas en su domicilio.

En nuestra opinión, las HBPM como grupo deben sustituir a las HNF en el tratamiento de las TVP. Probablemente ocurra lo mismo con el tromboembolismo pulmonar, aunque existen menos estudios al respecto $(13,22,23)$. Igualmente las HBPM están sustituyendo a las HNF en otras indicaciones de estas últimas, como son la cardiopatía isquémica inestable $(24,25,30)$, etc. Incluso se están usando sin complicaciones en las prótesis valvulares mecánicas que requieren la suspensión de la anticoagulación oral por algún procedimiento diagnóstico o terapéutico, o cuando está contraindicado el uso de la misma por diversos motivos $(24,30)$. En este sentido, el uso de la heparina sódica probablemente deba reservarse para situaciones muy concretas, e incluso llege a desaparecer. Es preciso que las autoridades sanitarias establezcan claramente todas las indicaciones de las HBPM para que se puedan usar sin problemas de tipo legal ya que sus beneficios son evidentes.

\section{Bibliografía}

1. Kearson C, Hirs J. Starting prophylaxis for venous thromboembolism postoperatively. Arch Intern Med 1995;155:366-71

2. Consensus Conference on antithrombotic therapy. Chest 1995; 108 (S): 225S-522S

3. Gabriel-Botella F, Lábios-Gómez M, Balaguer-Martínez JV, Fernández-López A. Prevención de la enfermedad tromboembólica venosa: heparinas no fraccionadas y heparinas de bajo peso molecular. Análisis de estas dos opciones. An Med Interna (Madrid) 1999; 16: 590-500.

4. Prandoni $\mathrm{P}$, Lensing A, Büller $\mathrm{H}$ et al. Comparison of subcutaneous low-molecular-weight heparin with intravenous standard heparin in proximal deep-vein thrombosis. Lancet 1992;339:441-5

5. Duroux P. A Collaborative European Multicentre Study. A randomized trial of subcutaneous low-molecular-weight heparin compared with intravenous unfractionated heparin in the treatment of deep-vein thrombosis. Throm Haemost 1991; 65(S):251-6

6. Lindmaker P, Holmström M, Granqvist S, Johnsson H, Lockner D. Comparison of once-daily subcutaneous Fragmin, with continuous intravenous unfractionated heparin in the treatment of deep-vein thrombosis. Throm Haemost 1994; 72(2): 186-90

7. Hull R, Raskob G, Pineo G et al. Subcutaneous low-molecular-weight heparin compared with continuos intravenous heparin in the treatment of proximal vein thrombosis. N Engl J Med 1992; 326: 975-82 
8. Hass S. Treatment of deep venous thrombosis and pulmonary embolism. Current recommendations. Med Clin N Am 1998;82:495-509

9. Bick R, Hass S. International consensus recommendations: summary statement and additional suggested guidelines. Med Clin N Am 1998;82:613-31

10. Ginsberg S. Management of venous thromboembolism. N Engl J Med 1996;335:1816-28

11. Pineo G, Hull R. Unfractionated and low-molecular-weight heparin in the treatment of venous thromboembolic disease. Med Clin N Am 1998;82:587-99

12. Litin S, Heit J, Mees K. Use of low-molecular-weight heparin in the treatment of venous thromboembolic disease. Answer to frequently asked questions. Mayo Clin Proc 1998;73:545-51

13. Simmoneau G, Sors H, Chambonnier B et al. A comparison of subcutaneous low-molecular-weight heparin with unfractionated heparin for acute pulmonary embolism. N Engl J Med 1997;337:663-9

14. Feissinger J, Lopez-Fernández M, Gatterer E et al. Once daily subcutaneous dalteparin for the initial treatment of acute deep vein thrombosis. Throm Haemost 1996;76:195-9

15. Downing L, Streiter R, Kadell A, Wilke C, Greenfield L, Wakefield T. Low-dose low-molecular-weight heparin is anti-inflammatory during venous thrombosis. J Vasc Surg 1998;28(S):848-54

16. Levine M, Gent M, Hirsh J. et al. A comparison of low-molecularweight heparin administered primarily at home with unfractionated heparin administered in the hospital for proximal deep vein thrombosis, N Engl J Med 1996; 334:677-81

17. Koopman M, Prandoni P, Piovella F. et al. Treatment of venous thrombosis with intravenous unfractionated heparin administered in the hospital as compared with subcutaneous low-molecular-weight heparin administered at home. The Tasman Study Group. N Engl J Med 1996;334:682-7

18. Harrison L, Mc Ginnis J, Crowther M, Ginsberg J, Hish J. Assessment of outpatient treatment of deep-vein thrombosis with low-molecularweight heparin. Arch Intern Med 1998;158:2001-3

19. Wells P, Kovacs M, Bormanis J. et al. Expanding eligibility for outpatient treatment of deep venous thrombosis and pulmonary embolism with low-molecular-weight heparin: a comparison of patient self-injection with homecare injection. Arch Intern Med 1998;158:1809-12
20. Van der Belt A, Bossuyt P, Prins M, Gallus A, Buller H. Replacing inpatient care by outpatient care in the treatment of deep venous thrombosis: an economic evaluation. Throm Haemost 1998; 79: 259-63

21. Hull R, Raskob G, Rosenbloom D. et al. Treatment of proximal vein thrombosis with subcutaneous low-molecular-weigh heparin versus intravenous heparin. An economic perspective. Arch Intern Med 1997; 157:289-94

22. Thery D, Simonneau G, Meyer G et al. Randomized trial of subcutaneous low-molecular-weight heparin CY 216 (Fraxiparine, ) compared with intravenous heparin in the curative treatment of submasive pulmonary embolism: a dose-ransing study. Circulation 1992; 85: 1380

23. Meyer G, Brenot F, Pacowet g et al. Subcutaneous low-molecularweight heparin Fragmin, versus intravenous unfractionated heparin in the treatment of acute non-massive pulmonary embolism: an open randomized pilot study. Throm Haemost 1995;74:1432

24. Gurfinkel E, Manos E, Mejail R et al. Low molecular heparin versus regular heparin or aspirin in the treatment of unstable angina and silent ischemia. J Am Coll Cardiol 1995;26:313-8

25. Turpie A. Low-molecular-weight heparins and unstable angina. Current perspectives. Haemostasis 1997;27(S1):19-24

26. Monreal M, Lafoz E, Olivé A, del Río L, Vedia C. Comparison of subcutaneous unfractionated heparin with a low molecular weight heparin (Fragmin) in patients with venous thromboembolism and contraindications to coumarin. Throm Haemost 1994;71:7-11

27. Van den Belt AGM, Prins MH, Lensing AWA, Castro AA, Clark OAC, Atallah AN, Burihan E. fixed dose subcutaneous low molecular weight heparins versus adjusted dose unfractionated heparin for venous thromboembolis (Cochrane Review). In: The Cochrane Library, Issue 1, 1999. Oxford: Update Software.

28. Barosi G, Marchetti M, Dazzi L, Quaglini S. Testing for occult cancer in patients with idiopathic deep vein thrombosis. A decision analysis. Thromb Haemost 1997;78:1319-26

29. Sorensen H, Mellemkjaer L, Steffensen F, Olsen J, Nielsen G. The risk of a diagnosis of cancer after primary deep venous thrombosis or pulmonary embolism. N Engl J Med 1998;338:1169-73

30. Aguilar D, Goldhaber S. Clinical uses of low-molecular-weight heparins. Chest 1999;115:1418-23 